\title{
Indicadores de sustentabilidad político-jurídica en la gestión de sitios patrimoniales interjurisdiccionales. Una aproximación metodológica
}

\author{
Political-legal sustainability indicators: methodological approaches \\ for the management of inter-jurisdictional heritage sites
}

\section{Norma Elizabeth Levrand* y Laura Zulaica**}

\footnotetext{
* Doctora en Derecho (Univ. Nacional del Litoral, UNL). Especialista en derecho laboral. Investigadora asistente del Instituto de Estudios Sociales (CONICET-UNER). Docente adjunta de la Facultad de Ciencias de la Gestión (Univ. Autónoma de Entre Ríos). Docente de la Facultad de Ingeniería y Ciencias Hídricas (UNL).

$\bowtie$ normalevrand@gmail.com https://orcid.org/0000-00021396-3688

** Doctora en Geografía. Lic. y magíster en Gestión Ambiental. Investigadora adjunta del CONICET, Instituto del Hábitat y del Ambiente, FAUD, Univ. Nacional de Mar del Plata (UNMdP). Docente en la FAUD y en la Fac. de Humanidades de la UNMdP. $\triangle$ laurazulaica@conicet.gov.ar https://orcid.org/0000-00018101-5957
}

RECIBID 0: 28.8.2020

\section{Resumen}

En los últimos tiempos, el lugar del desarrollo sustentable en la gestión del patrimonio natural y cultural ha sido una temática de creciente interés. Numerosos documentos destacan el importante rol del patrimonio en procesos hacia la sustentabilidad. Sin embargo, la articulación entre sustentabilidad y patrimonio es relativamente incipiente, como reciente es esta noción del desarrollo en la agenda patrimonial. En este trabajo se procura delinear un conjunto de indicadores de sustentabilidad jurídico-política para la gestión de bienes que han sido patrimonializados en Argentina y cuyo proceso involucra más de una jurisdicción. La sustentabilidad jurídico-política hace hincapié en las libertades de los actores, en la incorporación de miradas diversas, en la capacidad para expresarse y tomar decisiones en un contexto de fortalecimiento de los procesos democráticos. A partir de estudios anteriores que seleccionaron y construyeron indicadores aplicables al patrimonio cultural y natural y de la revisión de dos casos, se definen cuatro categorías que incluyen indicadores factibles de evaluar mediante un abordaje cualicuantitativo. La investigación aporta un instrumento metodológico que permite juzgar la eficiencia de la gestión de sitios patrimonializados, particularmente cuando involucran más de una jurisdicción. 
Palabras clave: patrimonio natural, patrimonio cultural, desarrollo sostenible, evaluación del impacto ambiental, evaluación, gestión.

\section{Abstract}

In recent times, sustainable development in the management of natural and cultural heritage has been a topic of growing interest. Numerous documents highlight the important role of heritage in processes towards sustainability. However, the articulation between sustainability and heritage is relatively incipient as recent is this notion of development in the heritage agenda. This paper seeks to outline a set of legal-political sustainability indicators for the management of assets that have become part of Argentina's heritage and that involved more than one jurisdiction along the process. Legal-political sustainability emphasizes the actors' freedom, entailing the incorporation of diverse perspectives, the ability to express themselves and make decisions, in a context of strengthening democratic processes. Based on previous studies that selected and built indicators applicable to cultural and natural heritage and the review of two cases, four categories are defined. Each one includes indicators that can be evaluated from a qualitative-quantitative approach. The research provides a methodological instrument to assess the efficiency of heritage management, particularly when they involve more than one jurisdiction.

Keywords: natural heritage; cultural heritage, sustainable development, environmental impact assessment, evaluation, management.

\section{Introducción}

La evaluación de la sustentabilidad constituye una herramienta cada vez más utilizada para definir alcances y medir progresos que permitan avanzar hacia los objetivos ecológicos, sociales, económicos y político-jurídicos del desarrollo sustentable. Mori y Christodoulou (2012) mencionan que el objetivo central de la evaluación de la sustentabilidad es proporcionar a los responsables de la toma de decisiones una valoración de los sistemas globales y locales que expresan la integración entre la naturaleza y la sociedad a corto y largo plazo, a fin de definir acciones para alcanzar una sociedad sustentable.

Desde el punto de vista conceptual, el desarrollo sustentable busca establecer un equilibrio entre las necesidades de las generaciones actuales y las futuras. A partir del Informe de la Comisión Brundtland (World Commission on Environment and Development [WCED], 1987), los distintos países han comenzado a definir objetivos y prioridades para alcanzar la sustentabilidad del desarrollo, considerando sus necesidades y aspiraciones nacionales. Si bien el concepto ha sido aceptado y difundido internacional- 
mente, también ha recibido críticas, dado que es usado con diversos enfoques y ha adquirido significados a veces ambiguos, que impiden el desarrollo de políticas concretas (Reboratti, 2000; Guimarães, 2003).

Para tratar de superar esas dificultades, numerosos autores han hecho un esfuerzo por sintetizar las características inherentes al desarrollo sustentable. Las dimensiones consideradas pueden resumirse en cuatro: ecológica, económica, sociocultural y política (Guimarães, 2003; Mori y Christodoulou, 2012), que se considera importante evaluar desde una perspectiva integral.

Las herramientas para evaluar la sustentabilidad son numerosas; entre ellas, los indicadores e índices desempeñan un papel relevante en el diagnóstico de los procesos enmarcados en principios de sustentabilidad.

Los esfuerzos de las organizaciones nacionales e internacionales por desarrollar modelos de indicadores e índices para evaluar y medir las dimensiones del desarrollo sustentable recibieron gran impulso tras la adopción de la Agenda 21, en la Cumbre de la Tierra de 1992 (United Nations, 1992). El capítulo 40 de la Agenda solicita específicamente a los países y organizaciones gubernamentales y no gubernamentales internacionales la adopción de indicadores de sostenibilidad aplicables a distintos ámbitos y escalas territoriales (Kwatra et al., 2016).

En el ámbito internacional, la evaluación de metas de sustentabilidad mediante indicadores se imprime en los 17 Objetivos de Desarrollo Sostenible (ODS), propuestos en la Cumbre para el Desarrollo Sostenible celebrada en Nueva York en 2015. Los Estados miembros de la Organización de las Naciones Unidas (ONU) aprobaron en dicha cumbre la Agenda 2030 para el Desarrollo Sostenible, que pretende disminuir la pobreza, la desigualdad, la injusticia y generar iniciativas para enfrentar el cambio climático.

En este marco, la evaluación de la sustentabilidad mediante indicadores atraviesa distintas áreas y contextos de gestión, uno de los cuales es el patrimonio. En los últimos tiempos, el lugar del desarrollo sustentable en la gestión del patrimonio natural y cultural ha sido una temática de interés creciente, manifiesta en documentos que destacan el importante rol del patrimonio en procesos de desarrollo sustentable. Algunas investigaciones recientes han elaborado categorías e indicadores para medir la sustentabilidad del patrimonio en términos económicos (Toselli et al., 2020), turísticos (Weng et al., 2019), e incluso han ensayado análisis multidimensionales (Nocca, 2017). Sin embargo, la articulación entre sustentabilidad y patrimonio es relativamente incipiente, y bastante reciente esta noción del desarrollo en la agenda patrimonial. En ese sentido, Cantar et al. (2021) analizan la vinculación entre sustentabilidad y patrimonio mediante la identificación de seis etapas, de 1960 a la actualidad, que culminan con la incorporación de la cultura y el patrimonio en los ODS de la Agenda 2030.

En la Agenda 2030, el ODS 11 propone que «las ciudades y los asentamientos humanos sean inclusivos, seguros, resilientes y sostenibles» y en la cuarta meta de este 
ODS se exige «redoblar los esfuerzos para proteger y salvaguardar el patrimonio cultural y natural del mundo» (11.4). En función de ello, la necesidad de construir y evaluar indicadores de sustentabilidad en sus distintas dimensiones se torna cada vez más relevante en la gestión de bienes que han sido patrimonializados. En particular, interesa evaluar aquellos aspectos referidos a la sustentabilidad jurídico-política del patrimonio natural y cultural.

En este sentido, la sustentabilidad jurídico-política hace hincapié en las libertades de los actores, en la incorporación de miradas diversas, en la capacidad para expresarse y tomar decisiones en un contexto de fortalecimiento de los procesos democráticos. Así, el fundamento político-jurídico de la sustentabilidad se encuentra estrechamente vinculado al proceso de profundización de la democracia y de construcción de la ciudadanía.

García y Priotto (2008) destacan que entre los objetivos de esta dimensión de la sustentabilidad se encuentra el fortalecimiento de las organizaciones sociales y comunitarias, la redistribución de activos y de información hacia los distintos sectores, el incremento de la capacidad de análisis de sus organizaciones y la capacitación para la toma de decisiones, la apertura del aparato estatal al control ciudadano y la incorporación del concepto de responsabilidad política en la actividad pública, entre otras cuestiones.

A partir de lo expuesto, en este trabajo se procura delinear un conjunto de indicadores de sustentabilidad jurídico-política para la gestión de bienes que han sido patrimonializados en Argentina y que involucran más de una jurisdicción en el proceso. Para enunciar y definir estos indicadores sirve de fundamento el antecedente de dos casos en los cuales la gestión se ve permeada por más de una jurisdicción: la quebrada de Humahuaca, declarada Patrimonio Mundial en la categoría Paisaje Cultural, y el delta del Paraná, territorio declarado Humedal de Importancia Internacional. En los casos mencionados, la problemática de la distribución de competencias se manifiesta notoriamente en la gestión de los bienes e incide en la sustentabilidad político-jurídica.

\section{Alcances metodológicos}

Fernández-Sánchez y Rodríguez-López (2010) señalan que uno de los mayores problemas para evaluar la sustentabilidad es identificar y seleccionar un conjunto de indicadores que permitan medirla. De acuerdo con Agol et al. (2014), la selección de indicadores es a menudo subjetiva y suele depender de factores como costos, facilidad de comprensión, confiabilidad y comparabilidad.

Con el objetivo de delinear indicadores pasibles de ser evaluados contemplando la dimensión político-jurídica de la sustentabilidad, se tomaron como base estudios antecedentes que implicaron la selección y construcción de indicadores aplicables al patrimonio 
natural y cultural (Álvarez Litben y Zulaica, 2015; Endere y Zulaica, 2015; Sánchez y Zulaica, 2018). Partiendo de lo anterior y de la revisión de dos casos antecedentes (Levrand, $2017 ; 2020$ ), se formuló un conjunto de preguntas que orientaron la construcción de indicadores evaluables utilizando escalas cualicuantitativas de valoración.

\section{Marco jurídico: competencias y patrimonialización}

La estructura federal argentina se organiza siguiendo tres principios: la supremacía del orden jurídico federal, la participación de las provincias en la formación de la voluntad federal y la distribución de competencias dentro del nivel nacional (Bidegaín, 2001).

Esta distribución de competencias puede identificarse a partir de las actividades propias (funciones) de cada uno de los poderes del Estado (Ejecutivo, Legislativo o Judicial). En Argentina, esta distribución se entrelaza con la estructura federal; existe descentralización administrativa en el ámbito del Estado nacional (de carácter funcional) y también en el ámbito de cada provincia, ya sea con base territorial —como las comunas y municipios - o con carácter funcional.

En cuanto al ámbito material, la distribución de competencias legislativas se consagra en el artículo 121 de la Constitución Nacional, que acuerda a las provincias todo el poder no delegado. No obstante, la flexibilidad de algunas normas constitucionales permite una actuación del Gobierno federal más extensa que la definida anteriormente. ${ }^{1}$

La doctrina jurídica argentina acuerda que, en virtud de que los recursos naturales pertenecen a las provincias, estas tienen la competencia legislativa sobre aquellos. No obstante, el artículo 41 de la Constitución Nacional establece que la Nación puede dictar normas de presupuestos mínimos de tutela del ambiente que sean obligatorias en todo el territorio argentino.

No existe un acuerdo similar en cuanto a las competencias legislativas en el ámbito cultural. En principio, cada jurisdicción (municipios, provincias y Nación) regula sobre los bienes culturales que son de su dominio o aquellos a los cuales se les reconoce valor en cada uno de los niveles estatales.

La gestión, como actividad funcional propia del Poder Ejecutivo, se compone de: a) una política pública, b) normas jurídicas que le den sustento y c) la administración (Brañes citado por Esaín, 2008, p. 400). En cuanto función del Poder Ejecutivo, la gestión ha sido desarrollada localmente, aunque en las últimas décadas se han producido dos

1 La propia Constitución incluye en el artículo 41, referido al derecho a un ambiente sano, la protección del patrimonio cultural. La discusión en el seno de la convención constituyente, como también la doctrina jurídica y judicial construida con posterioridad, confirmaron una concepción holística del ambiente, integrado por un componente natural y un componente cultural (Lorenzetti, 2008; Esaín, 2008; Levrand, 2015). 
desplazamientos contrapuestos. Por una parte, hacia el interior del Estado, se produjo una descentralización y una integración de la política ambiental y cultural; por otra, hacia el ámbito internacional, el Estado ha asumido compromisos de protección de estos bienes.

La patrimonialización de bienes naturales y culturales mediante su incorporación en listas elaboradas por organismos internacionales proyecta sobre el ordenamiento nacional particulares consecuencias en cuanto a la gestión de dichos bienes. Esto, junto con la textura abierta de muchas normas constitucionales y una dinámica del federalismo que se ha modificado a partir de la centralización de los poderes delegados y del cambio de la infraestructura socioeconómica (Frías, 2011), demanda el empeño de directivas de interpretación acordes a cada caso concreto.

De este modo, a la competencia provincial en materia de recursos naturales y bienes culturales locales debe adicionarse la competencia nacional en materia ambiental (leyes de presupuestos mínimos). En el ámbito de la ejecución administrativa, es importante recordar que el Estado nacional es responsable internacionalmente por las obligaciones contraídas en tratados e instrumentos de tal jerarquía y, conforme a ello, también será órgano administrativo fiscalizador del cumplimiento de estas normas en los ámbitos subnacionales.

En este aspecto, la complejidad de gestionar bienes que pertenecen territorialmente a más de una jurisdicción, o en los cuales existe una política pública de concertación entre diferentes niveles estatales, es bastante habitual. La distribución de competencias descrita se replica en las provincias, lo que multiplica la complejidad al momento de afrontar problemas transmunicipales (Rosatti, 2010).

En los casos que fundamentan este trabajo, la problemática de la distribución de competencias se manifiesta notoriamente en la gestión de los bienes declarados (figura 1). Se visibilizan de este modo diversas formas de organizar administrativamente la gestión. En algunos casos, esta responsabilidad la asumen los niveles provinciales y, en otros, organismos específicos que procuran articular los distintos niveles interesados.

La gestión interjurisdiccional de sitios naturales o culturales trasluce tensiones económicas, políticas y de poder que no siempre atañen a los valores culturales o naturales que se procura conservar. Delinear indicadores que permitan alcanzar las metas previstas en los ODS constituye una estrategia clave para evaluar la gestión de estos sitios en términos de sustentabilidad político-jurídica. 
Figura 1. Competencias y patrimonialización: esquema de síntesis

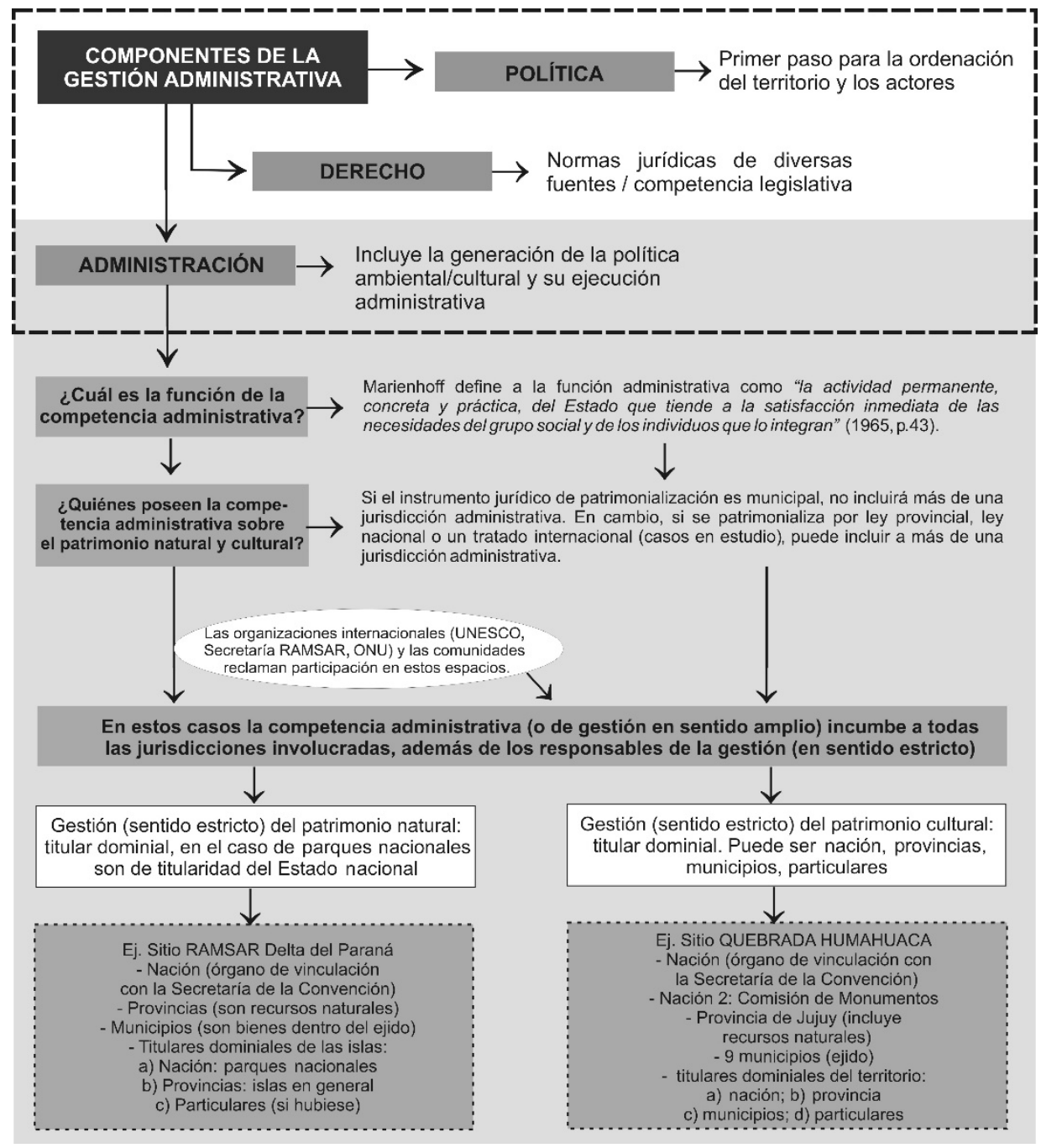

Fuente: Elaboración propia. 


\section{Los casos antecedentes}

Como se indicó en la introducción, dos casos sirven de antecedente para proponer un conjunto de indicadores de sustentabilidad político-jurídica: la quebrada de $\mathrm{Hu}$ mahuaca y el sitio Ramsar delta del Paraná (figura 2).

Figura 2. Localización de los casos antecedentes

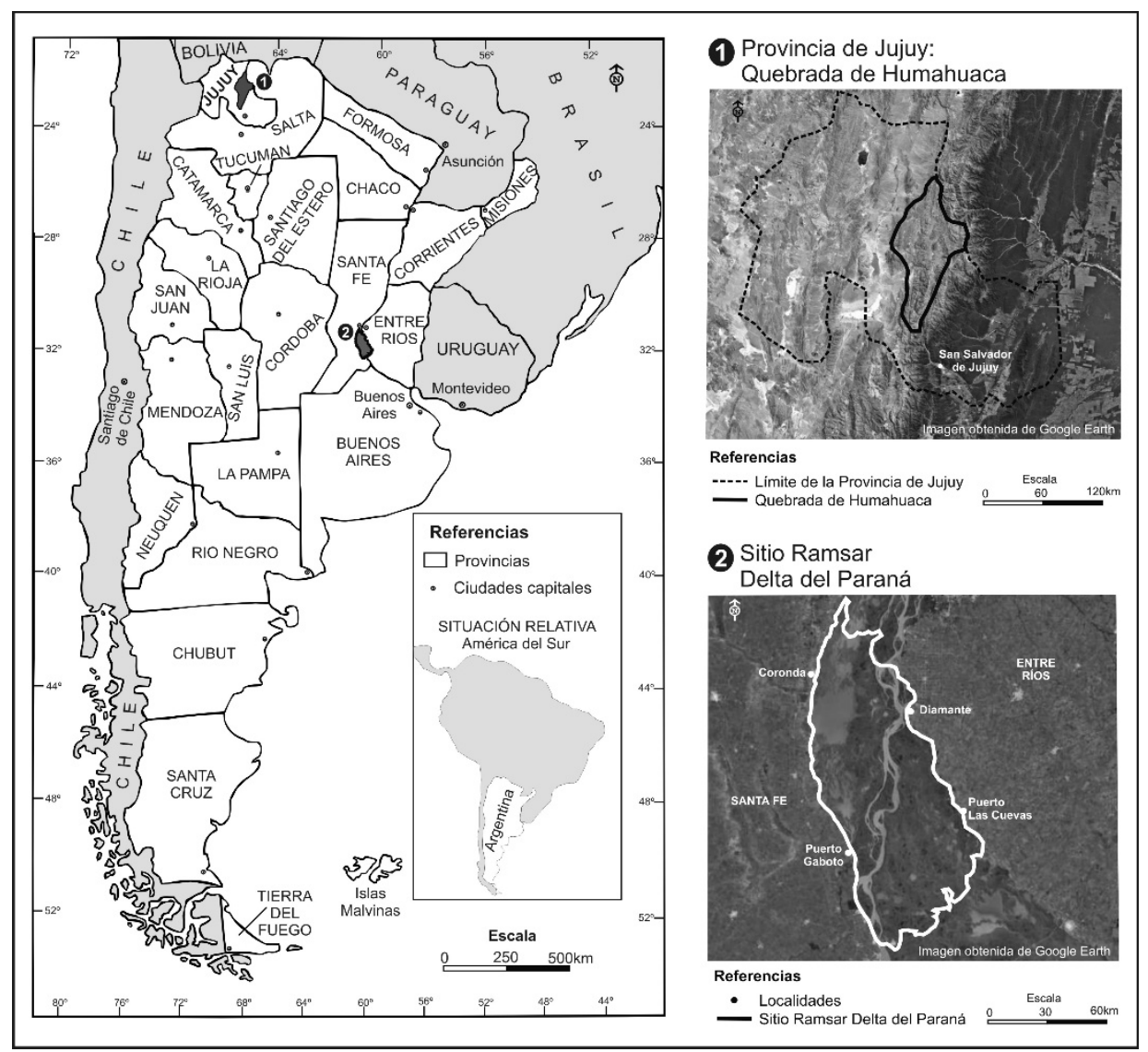

Fuente: Elaboración propia. 


\section{La quebrada de Humahuaca}

La inscripción de la quebrada de Humahuaca en la Lista del Patrimonio Mundial ${ }^{2}$ en 2003 incluyó 155 kilómetros de un estrecho y árido valle montañoso en el cual corre el río Grande, ubicado en la provincia de Jujuy, Argentina. La mayor parte de sus casi 35.000 habitantes (Instituto Nacional de Estadística y Censos [INDEC], 2010) reside en los pueblos más importantes, como Maimará, Tilcara y Humahuaca, mientras el resto ocupa poblados más pequeños y áreas rurales. Este paisaje cultural conserva casi intacto su entorno natural y posee vestigios arqueológicos, arquitectónicos e inmateriales que testimonian 10.000 años de historia.

La gestión del sitio congrega nueve comunas y municipios, la provincia de Jujuy, la Comisión Nacional de Monumentos, de Lugares y de Bienes Históricos y la Comisión Nacional para la Cooperación con la UNESCO, que funciona en el ámbito de Cancillería de la Nación.

En 2003 se creó la Unidad de Gestión Quebrada de Humahuaca, dependencia de la Dirección Provincial de Patrimonio, a cargo de la Secretaría de Turismo y Cultura. Su objetivo era preservar y administrar el sitio en forma eficiente en el marco de un desarrollo económico sustentable, el respeto por la identidad, la visión y los intereses de la comunidad local con el propósito de la apropiación y conservación del paisaje.

Durante el proceso de postulación, los habitantes del territorio quebradeño comenzaron a plantear dudas e inquietudes respecto del procedimiento y de los fines de la postulación y a exigir una participación activa en el proceso. Estos mecanismos de participación se formalizaron a partir de la creación de las comisiones locales de sitio (una por cada municipio o comuna) como un «actor institucional». A partir de esta institucionalización, la Unidad de Gestión conforma un nexo entre las comisiones locales de sitio, que representan de modo directo los intereses de las comunidades humahuaqueñas y otros organismos del nivel provincial.

En síntesis, la gestión de este sitio patrimonial se encuentra a cargo de un organismo provincial (Unidad de Gestión Quebrada de Humahuaca) que opera como articulador de las instituciones de participación directa (comisiones locales de sitio) y los municipios y comunas con los organismos nacionales (Comisión Nacional de Monumentos, de Lugares y de Bienes Históricos y Comisión Nacional para la Cooperación con la UNESCO). 


\section{El sitio Ramsar delta del Paraná}

En 2016 el humedal delta del Paraná fue inscripto en la Lista de Humedales de Importancia Internacional de la Convención Ramsar. ${ }^{3}$ Este sitio comprende un territorio de aproximadamente 240.000 hectáreas localizadas en la zona de islas de las provincias de Entre Ríos (departamento Diamante) y Santa Fe (departamento San Jerónimo) e incluye dos parques nacionales.

En la gestión del sitio coinciden tres jurisdicciones: la Nación, a través de la Administración de Parques Nacionales, y las provincias de Entre Ríos y Santa Fe. Asimismo, en el entorno del sitio se encuentran varios municipios y comunas de ambas provincias. En virtud de la multiplicidad jurisdiccional y administrativa sobre el territorio, en junio de 2017 se conformó el Comité Interjurisdiccional de Manejo (en adelante CIM), en el cual intervienen, además de los gobiernos de las jurisdicciones mencionadas, los gobiernos municipales, el Instituto Nacional de Tecnología Agropecuaria (INTA), varias instituciones académicas y algunas ONG.

Hacia mediados de 2018, el proceso de desarrollo de un plan de manejo del sitio y la implementación de comisiones técnicas que involucran actores con anclaje territorial en el CIM favorece la ampliación de agentes que participan en las discusiones producidas en su seno. Entre ellos pueden mencionarse ONG que no se encontraban vinculadas anteriormente, algunos pobladores de las ciudades y localidades ribereñas, pobladores y usuarios de las islas, emprendedores turísticos, entre otros.

De este modo, el CIM es un espacio de concertación de múltiples intereses, aunque, conforme a su reglamento, las opiniones y los debates no son vinculantes para las administraciones provinciales ni la nacional.

\section{Definición de indicadores de sustentabilidad político-jurídica}

En términos generales, y desde el punto de vista operativo, un indicador puede definirse como una variable que describe características del estado de un sistema a través de datos observados o estimados. Desde el punto de vista conceptual, los indicadores contribuyen a aprender, entender y estructurar la definición de políticas y la interpretación de tendencias para encontrar soluciones a problemas clave que inciden en la sustentabilidad, en este caso jurídico-política. Tradicionalmente, y siguiendo a Holden 
(2013), los indicadores de sustentabilidad pueden dividirse en dos grupos opuestos: aquellos centrados en un enfoque «técnico» $\mathrm{u}$ «orientado a expertos» y los que asumen un enfoque «participativo» u «orientado al ciudadano». Más recientemente, varios autores han argumentado la convergencia teórica y práctica de estos dos enfoques (Reed et al., 2006; Rametsteiner et al., 2011).

En esta primera aproximación a la definición de indicadores de sustentabilidad político-jurídica, sin desconocer los beneficios de la aplicación de modelos participativos e integrados, se asume principalmente un enfoque técnico, con la expectativa de generar una base que permita abrir la discusión entre distintos actores. Desde esta perspectiva, los indicadores son instrumentos indispensables para recopilar información que sirva para planificar, implementar y evaluar políticas de desarrollo sustentable a través del tiempo (Singh et al., 2009; Moreno-Pires y Fidélis, 2012). Además, constituyen una herramienta de evaluación comparativa que puede ser ajustada y mejorada.

El análisis de los casos citados y la formulación de preguntas sobre los aspectos relevantes en la evaluación de la sustentabilidad política y jurídica de la gestión derivaron en la definición de un conjunto de indicadores que puede dividirse en cuatro categorías principales: 1) administración de los bienes patrimonializados; 2) conformación de los comités de gestión (designación que procura unificar los diversos nombres de estos organismos); 3) participación de expertos, y 4) integración de organizaciones de la sociedad civil (OSC) y de los ciudadanos (tabla 1).

La categoría 1 (administración de los bienes patrimonializados) procura considerar el grado de institucionalidad y formalización jurídica de los organismos de gestión de los sitios y la articulación establecida entre distintos niveles jurisdiccionales. Teniendo en cuenta que los casos que constituyen antecedentes de este trabajo presentan una gestión interjurisdiccional, la formalización de acuerdos entre las jurisdicciones es relevante para evaluar la sostenibilidad de la gestión. Las facultades y obligaciones de cada una de las administraciones involucradas pueden manifestarse en los acuerdos de conformación de comités de gestión o surgir de acuerdos interjurisdiccionales.

En una modalidad, estos acuerdos pueden manifestarse como convenios interjurisdiccionales internos, entendidos como acuerdos entre la Nación y las provincias que armonizan la programación, la ejecución o el control de una medida política o administrativa en competencias concurrentes (Esaín, 2008). Otra modalidad posible es la actuación sincronizada a través de un reglamento conjunto. En este caso, las «distintas administraciones públicas se autolimitan, participativamente, estableciendo el conjunto de reglas inferidas de sus sistemas jurídicos que regularán la actuación sincronizada que deben desplegar para atender el caso multidimensional de base constitucional o supralegal» (Reyna, 2011).

El segundo indicador relevante se refiere a la sostenibilidad económica de la gestión conjunta. En este punto pueden existir pautas de manejo de los planes conjuntos 
entre administradores. Otra posibilidad es la creación de un fondo común para la gestión del sitio, como también que las administraciones involucradas atribuyan partidas y determinen el destino de los fondos generados por el propio sitio a partir, por ejemplo, del producido de las visitas.

Tabla 1. Propuesta de indicadores para evaluar la sustentabilidad político-jurídica en la gestión de sitios patrimoniales interjurisdiccionales

\section{1) Administración de los bienes patrimonializados}

Distribución de facultades/obligaciones de los administradores

Distribución de los recursos entre los administradores

Nivel de participación de las mujeres en los grupos administradores

Grado de integración vertical entre los organismos de gobierno

Grado de integración horizontal entre los organismos de gobierno

\section{2) Conformación de los comités de gestión}

Instancia de formalización del comité de gestión

Representatividad de los miembros que integran el comité de gestión

Nivel participación de las mujeres en el comité de gestión

Frecuencia de las reuniones

Capacidad de organización de las reuniones

Nivel de participación de los miembros en las reuniones

Capacidad para tomar decisiones concretas

Capacidad de organización flexible en función de temas de interés específico

Capacidad de convocatoria a nuevos miembros

\section{3) Participación de expertos}

Involucramiento de expertos en los procesos de gestión

Capacidad de toma de decisiones de los expertos

Nivel de participación de las mujeres entre los expertos

\section{4) Integración de organizaciones de la sociedad civil y de los ciudadanos}

Existencia y capacidad organizativa de OSC

Capacidad de participar en la toma de decisiones

Instancias de participación ciudadana incluyendo la diversidad de grupos sociales

Instancias de participación directa de la ciudadanía en la toma de decisiones

Grado de involucramiento de las comunidades

Fuente: Elaboración propia sobre la base de estudios antecedentes y revisión de los casos. 
Considerar el nivel de participación de las mujeres en todos los ámbitos estudiados es relevante para evaluar la igualdad de género. Si bien acordamos con una definición de género como construcción sociocultural que diferencia roles y, por ende, con la idea de que existen tantos géneros como autopercepciones al respecto, a los fines de este trabajo limitamos la consideración a varones y mujeres. En este punto en particular, evaluar el lugar de las mujeres puede evidenciar los obstáculos que estas enfrentan para llegar a posiciones de decisión.

Las responsabilidades de cada nivel de gobierno sobre el sitio se verifican en su grado de participación en las acciones relativas a este. La complejidad en la distribución de competencias suele obstaculizar acciones articuladas que favorezcan la sustentabilidad de la gestión. En el caso del sitio delta del Paraná, en el CIM confluyen representantes de distintos niveles estatales capaces de coordinar acciones conjuntas. Sin embargo, pueden surgir dificultades atinentes a la competencia material de cada uno.

La situación mencionada exige considerar la distribución y la articulación de competencias en el nivel horizontal del gobierno. La fuente para evaluar este indicador no es evidente; requiere indagar en planes conjuntos y en la percepción de la ciudadanía y los usuarios del sitio acerca de las normas que pueden solaparse entre distintos organismos de gobierno. También puede requerir la inclusión de diversos agentes públicos en los comités de gestión. Los indicadores de este grupo se describen sintéticamente en la figura 3.

Figura 3. Descripción de los indicadores propuestos para la administración de los bienes patrimonializados

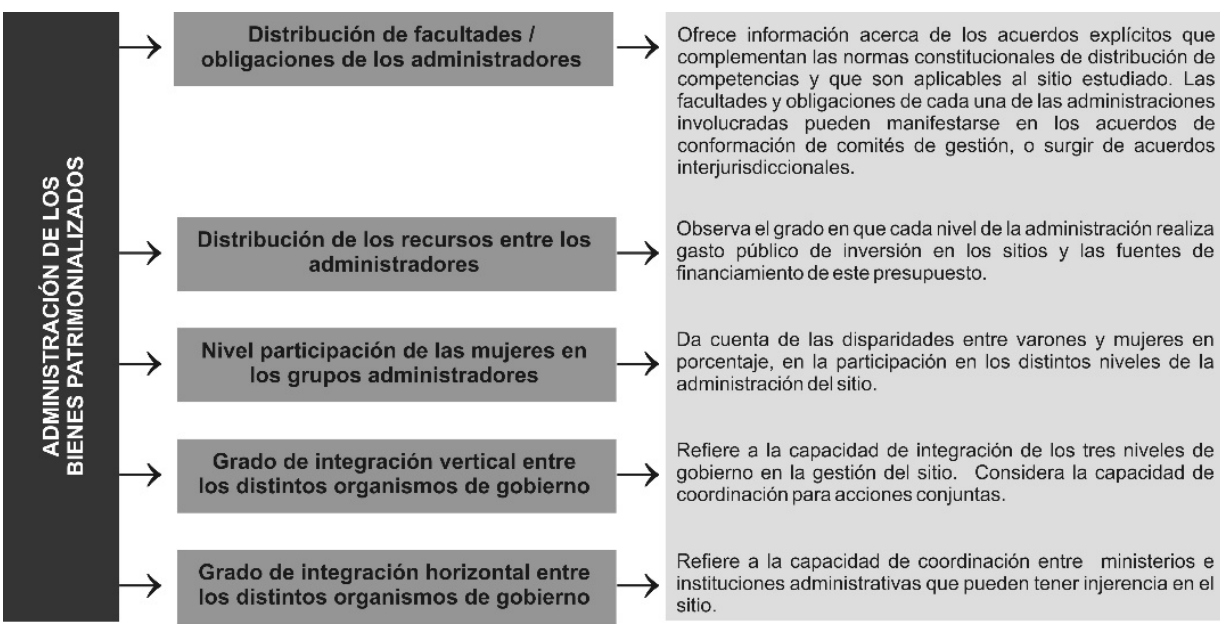

Fuente: Elaboración propia. 
La categoría 2 (conformación de los comités de gestión) es la más robusta, dado que estas instituciones se generan especialmente para administrar sitios con valor patrimonial o natural que involucran a más de una jurisdicción.

El primer eje se refiere a la formalización jurídica de los comités de gestión, que puede realizarse por un instrumento jurídico o de hecho (en estos casos suelen adoptar la denominación de redes, multisectoriales e incluso asambleas). La instrumentación jurídica no implica efectividad. En los antecedentes que fundamentan este trabajo, ambos comités de gestión se encuentran formalizados y desarrollan sus actividades conforme a sus reglamentos. En estos casos, es relevante dar cuenta de su conformación y su articulación con otros organismos estatales del mismo o de distinto nivel jurisdiccional.

En cuanto a la conformación, resulta pertinente considerar si los representantes son designados mediante la participación directa de la ciudadanía que habita esos territorios, por las autoridades gubernamentales o por interés de los participantes en integrar el comité. A menudo será necesario investigar el grado en que quienes integran el comité pueden tomar decisiones vinculantes sobre aspectos del sitio y llevar problemáticas relativas a los intereses sobre el sitio a la discusión del comité. También es necesario evaluar el grado de vinculación del integrante con el sitio (por ejemplo, el gobernador es legítimo representante del pueblo, pero seguramente tiene muchas prioridades antes que el sitio natural/cultural). En los sitios analizados, la situación es muy diversa: en la quebrada de Humahuaca se ha previsto la participación de la ciudadanía a partir de representantes elegidos directamente; en el delta del Paraná la participación es institucional y recae en personas que poseen interés en las acciones sobre el sitio.

Para completar el eje jurídico, debe considerarse el carácter vinculante o no de las decisiones del comité de gestión para las autoridades. En ninguno de los casos analizados las decisiones tienen carácter vinculante; sin embargo, podrían evaluarse otras medidas de acatamiento, como acuerdos con las autoridades para resolver conflictos presentes en el sitio, adopción de las decisiones por las autoridades, etcétera.

El segundo eje a evaluar se refiere a aspectos procesales de las actividades del comité de gestión. Tanto la frecuencia de las reuniones como el lugar en el que se desarrollan (rotación de espacios, si están situados o no en el sitio, si son cercanos a las poblaciones, etcétera) evidencian la capacidad del comité de dar respuesta a problemas que surgen en el sitio y a involucrar a la ciudadanía en el gobierno de este.

Por otra parte, a partir de la conformación del comité podrá evaluarse su flexibilidad para adecuarse a nuevas problemáticas que surgen en el sitio y adecuar la participación de expertos y la ciudadanía para resolverlas. Las dimensiones se sintetizan en la figura 4. 
Figura 4. Descripción de los indicadores propuestos para la conformación de los comités de gestión

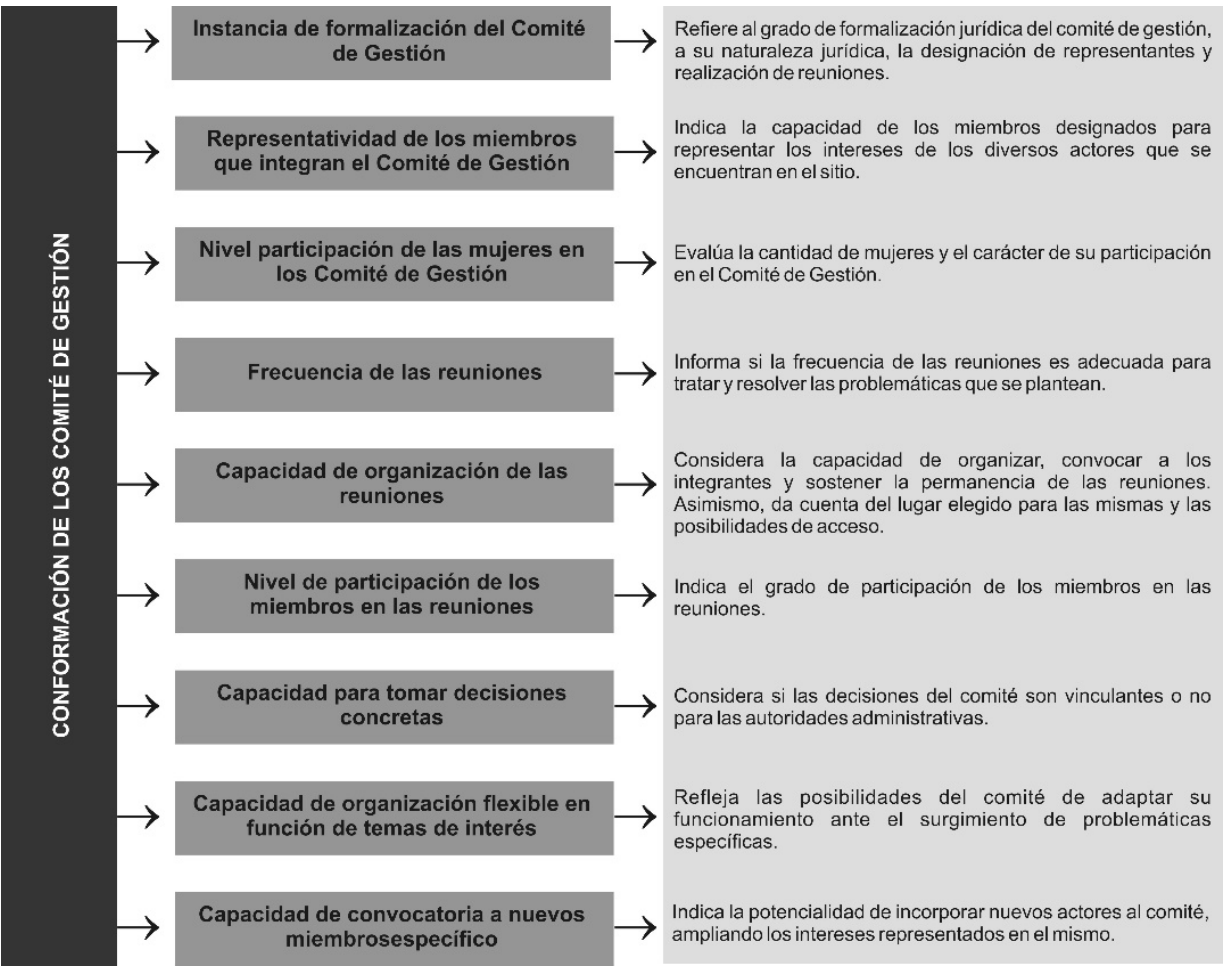

Fuente: Elaboración propia.

La tercera categoría se refiere a la participación de los expertos en estos comités de gestión. La relevancia de la actuación de los expertos, incrementada a partir de los años setenta, ha generado no solo un modelo alternativo de organización del saber, sino también una nueva manera de pensar el poder y el rol del conocimiento en la política (Jasanoff, 2004; Anheier, 2010). Así, la relevancia otorgada a estos agentes se fundamenta en que tanto la identificación como la construcción de las nociones de patrimonio cultural y natural provienen de una intermediación experta (Muriel, 2015). Es decir, el consenso científico que, desde distintas perspectivas disciplinarias, se exhibió respecto de los conceptos relevantes para gestionar el territorio ha dado forma a estas nociones y a su aplicación territorial (Nowotny, 2011). En este sentido, la comunidad científica tiene amplia incidencia en la gestión pública del ambiente y del patrimonio cultural, y por ello es relevante considerar su participación en los comités de gestión de los sitios. 
A partir de los casos analizados, se advierte que la participación de los expertos puede estar ligada a problemáticas que requieren una solución científica (aquellas ligadas a la conservación de ciertos materiales o al manejo de especies animales o vegetales exóticas, por ejemplo), como también a situaciones originadas por el impacto antrópico sobre el sitio. En estos últimos casos, la participación de expertos puede ser diferente si se limitan a ofrecer información científica para el debate en el seno de asambleas u órganos particulares o si se encuentran en puestos de toma de decisión.

Finalmente, la participación de mujeres expertas es una variable que debe ser considerada para evaluar la igualdad de género en los comités de gestión. La descripción de los indicadores propuestos se presenta en la figura 5.

Figura 5. Descripción de los indicadores propuestos para la participación de expertos

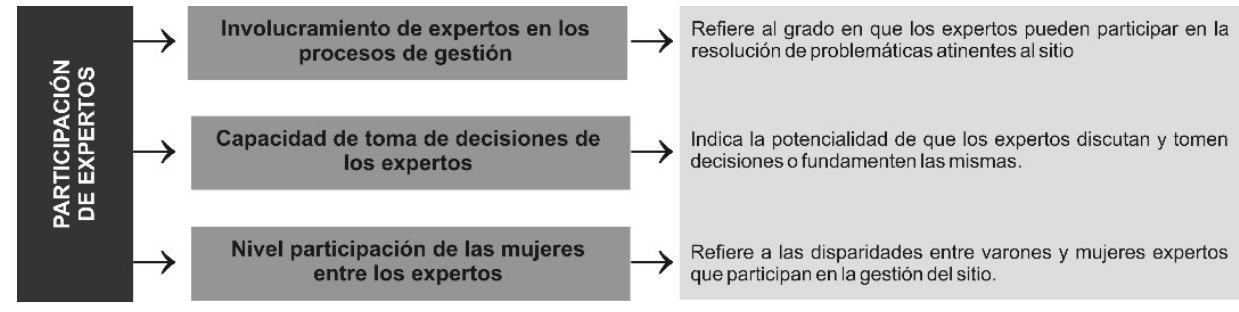

Fuente: Elaboración propia.

La cuarta categoría se refiere a la integración de la ciudadanía y de las organizaciones de la sociedad civil en los órganos de gestión y decisión de los sitios. Las posibilidades de habilitación jurídica de la participación en la gestión de sitios patrimoniales están determinadas por el ordenamiento jurídico y por la capacidad de institucionalizar la dimensión activa de la ciudadanía.

En los casos analizados se han identificado algunas dificultades para la participación de la ciudadanía en la gestión: en el delta del Paraná la declaración abarca territorio isleño, cuya población, escasa y dinámica, rota entre asentamientos ribereños y propiamente isleños; en la quebrada de Humahuaca se encuentran nueve ciudades o comunas, con una población de baja densidad, pero que incluye distintos intereses sobre el área.

Otra de las dificultades de la participación de la ciudadanía en la gestión refiere a identificar quiénes participarán en las reuniones de los órganos del comité de gestión y en qué carácter. Esto es particularmente problemático cuando existen diversos grupos sociales o comunidades autoafirmativas que poseen intereses diferentes sobre la gestión del sitio. A su vez, la posibilidad de participar en órganos de decisión o en órganos asamblearios implica distintas aperturas democráticas en la gestión de los sitios.

Por otra parte, la preocupación por la integración de los ciudadanos en las distintas etapas del proceso de gobernanza de los sitios patrimoniales tropieza con la limitación de 
«las energías participativas de los ciudadanos» (Font, 2004). Razones de tiempo y capacidades deliberativas limitan la participación de las comunidades en política.

Una de las propuestas que han encontrado buena recepción consiste en considerar a las OSC - tengan o no reconocimiento jurídico- como sujetos capaces de encauzar los intereses de sus miembros. En este caso, las limitaciones referidas a la perseverancia de la participación se sortean por la propia finalidad de estas organizaciones, y se fomenta asimismo una mayor representación de intereses divergentes sobre el área. Sin embargo, pueden surgir otras tensiones, referidas a la intervención de OSC de nivel nacional e internacional conjuntamente con otras locales, e incluso puede ocurrir, como en el caso del sitio Ramsar delta del Paraná, que ninguna de las organizaciones tenga domicilio en el sitio (el cual, recordemos, comprende territorio isleño). El reglamento cumple un rol fundamental para habilitar (o no) a las OSC a participar en la gestión.

De este modo, la existencia y la capacidad organizativa de las OSC da cuenta de su reconocimiento jurídico, atento a que su formalización permite una mayor injerencia en el ámbito público y, en algunos casos, permite que la organización represente intereses diversos sobre el sitio y movilice a más personas en él. En la figura 6 se describen los indicadores considerados.

Figura 6. Descripción de los indicadores propuestos para la integración de organizaciones de sociedad civil y de los ciudadanos

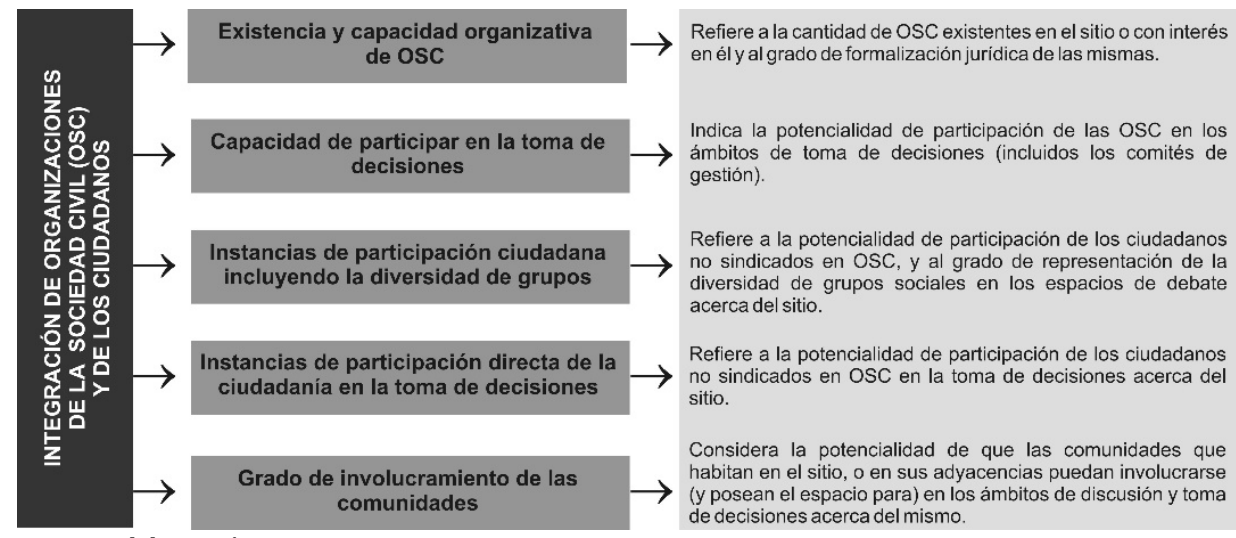

Fuente: Elaboración propia. 


\section{Consideraciones finales}

El desarrollo de indicadores se ha convertido en una tarea clave de los gobiernos para diagnosticar el estado de situación y generar propuestas tendientes a alcanzar la sustentabilidad en distintos ámbitos. En este trabajo se profundiza en la dimensión político-jurídica de la sustentabilidad asociada con la gestión de bienes patrimoniales naturales y culturales que presentan complejidad interjurisdiccional y se aportan herramientas analíticas e interpretativas útiles que conforman una base sólida para la formulación de políticas y la comunicación pública (Singh et al., 2009).

La definición de categorías cualicuantitativas permite establecer una estructura común de análisis que favorece la comparación de resultados obtenidos por cada una de ellas y con relación al total. La valoración de cada indicador permitirá definir índices sintéticos que resuman la información de cada grupo de indicadores y también del total. De esta manera, el desarrollo de índices permite simplificar la información y así facilita la comprensión e interpretación de los hallazgos (King, 2016).

Los índices que pueden construirse a partir de la integración de los indicadores propuestos conforman una herramienta para monitorear las tendencias de sustentabilidad a mediano y largo plazo. Asimismo, la información que sintetizan contribuye a definir escenarios de corto plazo y proponer decisiones hacia el futuro. Los indicadores integrados en índices son útiles para identificar problemas, establecer objetivos de desarrollo sustentable e identificar estrategias de gestión adecuadas.

El derecho argentino no posee reglas para la actuación conjunta de las diversas escalas de la administración. Sin embargo, las posibilidades de coordinación y articulación entre distintas jurisdicciones administrativas para la gestión del patrimonio y su institucionalización jurídica son diversas. En los casos que fundamentan este trabajo se han creado organismos multidimensionales y participativos que procuran abarcar los diversos intereses que operan sobre los sitios. La evaluación de su eficiencia en términos de la sustentabilidad de los sitios requiere la definición de instrumentos como los que se proponen en este trabajo.

Más allá de los beneficios mencionados en el uso de estos instrumentos, es importante destacar que categorías cualicuantitativas de valoración como las aquí propuestas asumen ciertos niveles de subjetividad, necesarios para ganar en significación, pero pueden generar pérdidas de especificidad respecto de los aspectos de la sustentabilidad que se están midiendo. Al respecto, validar los indicadores desde un enfoque participativo permitiría ajustar los alcances a fin de lograr una mayor representatividad de los aspectos que se pretenden evaluar. 


\section{Referencias bibliográficas}

Agol, D., Latawiec, A. E., y Strassburg, B. B. N. (2014). Evaluating impacts of development and conservation projects using sustainability indicators: Opportunities and challenges. Environmental Impact Assessment Review, 48, 1-9.

Álvarez Litben, S., y Zulaica, L. (2015). Indicadores de sustentabilidad en sistemas de albarradas: Aportes metodológicos. Revista Letras Verdes, 18, 184-207.

Anheier, H. (2010). Social science research outside the ivory tower: The role of thinktanks and civil society. En United Nations Educational, Scientifical an Cultural Organization e International Social Science Council, World social sciences report: Knowledge divides (pp. 338-341). París: UNESCO.

Bidegain, C. (2001). Curso de derecho constitucional: Tomo III. Buenos Aires: AbeledoPerrot.

Cantar, N., Endere M. L., y Zulaica, L. (2021). La «arqueología» de la sustentabilidad en la concepción del patrimonio cultural. Revista de Estudios Sociales, 75, 71-86.

Esaín, J. (2008). Competencias ambientales. Buenos Aires: Lexis Nexis.

Endere, M. L., y Zulaica, L. (2015). Sustentabilidad socio-cultural y buen vivir en sitios patrimoniales: Evaluación del caso Agua Blanca, Ecuador. Revista Ambiente \& Sociedade, 18(4), 265-290.

Fernández-Sánchez, G., y Rodríguez-López, F. (2010). A methodology to identify sustainability indicators in construction project management: Application to infrastructure projects in Spain. Ecological Indicators, 10, 1193-1201.

Font, J. (2004). Participación ciudadana y decisiones públicas: Conceptos, experiencias y metodologías. En A. Ziccardi (coord.), Participación ciudadana y políticas sociales en el ámbito local (pp. 23-42). México: UNAM.

Frias, G. (2011). Nuevos desafíos del federalismo. En A. Hernández y G. Barrera Buteler (coords.), Derecho público provincial. Buenos Aires: Abeledo-Perrot.

García, D., y Priotto, G. (2008). Módulo 2: La sustentabilidad como discurso ideológico. Buenos Aires: SAySD.

Guimarães, R. (2003). Tierra de sombras: Desafíos de la sustentabilidad y del desarrollo territorial y local ante la globalización corporativa. Santiago de Chile: CEPAL.

Holden, M. (2013). Sustainability indicator systems within urban governance: Usability analysis of sustainability indicator systems as boundary objects. Ecological Indicators, 32, 89-96.

Instituto Nacional de Estadística y Censos. (2010). Censo nacional de población, hogares y viviendas. Buenos Aires: INDEC.

Jasanoff, S. (2004). States or knowledge: The co-production of science and social order. Londres: Routledge. 
Kwatra, S., Kumar, A. Sharma, P., Sharma, S., y Singhal, S. (2016). Benchmarking sustainability using indicators: An Indian case study. Ecological Indicators, 61, 928-940.

King, L. O. (2016). Functional sustainability indicators. Ecological Indicators, 66, 121131.

Levrand, N. (2015). Normas de presupuestos mínimos de protección del patrimonio cultural en Argentina: ¿Posibles y vigentes? E-rph, (16), 2-19.

Levrand, N. (2017). La regulación del patrimonio cultural mundial en Argentina: Dominio, competencias y significaciones. (Tesis doctoral). Facultad de Ciencias Jurídicas y Sociales, Universidad Nacional del Litoral, Santa Fe.

Levrand, N. (2020). Federalismo de concertación y nuevas formas de gobierno de espacios interjurisdiccionales: El caso del sitio Ramsar Delta del Paraná. Derecho y Ciencias Sociales, (23), 93-110.

Lorenzetti, R. (2008). Teoría del derecho ambiental. Buenos Aires: La Ley.

Moreno-Pires, S., y Fidélis, T. (2012). A proposal to explore the role of sustainability indicators in local governance contexts: The case of Palmela, Portugal. Ecological Indicators, (23), 608-615.

Mori, K., y Christodoulou, A. (2012). Review of sustainability indices and indicators: Towards a new City Sustainability Index (CSI). Environmental Impact Assessment Review, 32(1), 94-106.

Muriel, D. (2015). La mediación experta en la construcción del patrimonio cultural como producción contemporánea de «lo nuestro». Revista de Antropología Iberoamericana, 10(2), 259-288.

Nocca, F. (2017). The role of cultural heritage in sustainable development: Multidimensional indicators as decision-making tool. Sustainability, 9(10), 1882.

Nowotny, H. (2011). ¿Salirse de la ciencia es salir de sincronía? En Informe sobre las ciencias sociales en el mundo: Las brechas del conocimiento (pp. 337-340). México: Foro Consultivo Científico y Tecnológico.

Rametsteiner, E., Pülzl, H., Alkan-Olsson, J., y Frederiksen, P. (2011). Sustainability indicator development-science or political negotiation? Ecological Indicators, 11, 61-70.

Reboratti, C. (2000). Ambiente y sociedad: Conceptos y relaciones. Buenos Aires: Planeta.

Reed, M. S., Fraser, E. D., y Dougill, A. J. (2006). An adaptive learning process for developing and applying sustainability indicators with local communities. Ecological Economics, 59(4), 406-418.

Reyna, J. (2011). Globalización, pluralidad sistémica y derecho administrativo: Apuntes para un derecho administrativo multidimensional. En F. Romeu, E. Gabardo, y D. Wunder Hachem (coords.), Globalização, direitos fundamentais e direito administrativo (pp. 25-53). Belo Horizonte: Fórum.

Rosatti, H. (2010). Tratado de derecho municipal: Tomo II. Santa Fe: Rubinzal Culzoni. 
Sánchez, L., y Zulaica, L. (2018). Ciudades vivas: Aportes mediante indicadores de sustentabilidad patrimonial residencial en aglomeraciones intermedias bonaerenses argentinas. Casos Mar del Plata y Tandil. Dearq, 23(2), 178-187.

Singh, R. K., Murty, H., Gupta, S., y Dikshit, A. (2009). An overview of sustainability assessment methodologies. Ecological Indicators, 9(2), 189-212.

Toselli, C., Takáts, A., y Traverso, L. (2020). Análisis de la sostenibilidad en emprendimientos turísticos ubicados en áreas rurales y naturales: Estudios de caso en la provincia de Entre Ríos, Argentina. Cuadernos de Turismo, (45), 461-489.

United Nations. (1992). Earth summit: Agenda 21. The United Nations Programme of Action from Rio. Nueva York: UN.

World Commission on Environment and Development. (1987). Our common future: The World Commission on Environment and Development. Oxford: Oxford University Press.

Weng, L., He, B. J., Liu, L., Li, C., y Zhang, X. (2019). Sustainability assessment of cultural heritage tourism: Case study of Pingyao ancient city in China. Sustainability, 11(5), 1392. 